\title{
Correspondance
}

\section{SSRI treatment for under-18s}

T et me see if I've got this straight. In$\checkmark$ dustry bias in reporting the results of trials of selective serotonin reuptake inhibitors (SSRIs) is probable, ${ }^{1}$ although an employee of a multinational drug company in another country doubts that this is so. ${ }^{2}$ Another therapist (the author of a book reviewed in CMAf) feels that mental illness has become far too commercialized (and the reviewer notes that, for pointing this out, the book's author has been personally maligned). ${ }^{3}$ Data are lacking because they are not known (because of a globally inadequate system for reporting adverse drug reactions ${ }^{4}$ ), are not available (because they have not been published ${ }^{5}$ ) or cannot be discussed by physicians who have engaged in nondisclosure contracts. ${ }^{6}$ SSRIs may be associated with an incidence of serious side effects (including withdrawals) of up to $25 \%$, and the placebo response rate can be as high as $40 \%$ to $60 \%$, although there may be a $70 \%$ response rate on some criteria of depression. ${ }^{6}$ There is no evidentiary basis to prescribe or not prescribe SSRIs in patients under 18 years of age, and, either way, all of these uses are "off label" for patients in this age group. Furthermore, no clear leadership position is evident among child psychiatrists, almost all of whom are aligned with this controversy in some way.

As a personal standard, I try never to complain without offering some constructive suggestion. Having perused a selection of the currently available world literature on this topic, my impression is that SSRIs should be used with caution in this age group, and only as a last resort, after the failure of all other obvious psychosocial and environmental interventions, and with close attention to symptoms of mood instability (serious adverse behavioural and emotional reactions including agitation, irritability, behavioural disinhibition and suicidality).

Mark A. Voysey

Adolescent Psychiatrist

Toronto, Ont.
References

1. Bhandari M, Busse JW, Jackowski D, Montori VM, Schünemann H, Sprague S, et al. Association between industry funding and statistically significant pro-industry findings in medical and surgical randomized trials. CMA7 2004;170(4): 477-80.

2. Hirsch L. Randomized clinical trials: What gets published, and when? [editorial]. CMA7 2004; 170(4):481-3.

3. Neilson S. Healy and Goliath [book review]. CMA7 2004;170(4):501-2.

4. Herxheimer A, Mintzes B. Antidepressants and adverse effects in young patients: uncovering the evidence [editorial]. CMA7 2004;170(4):487-9.

5. The "file drawer" phenomenon: suppressing clinical evidence [editorial]. CMA7 2004;170(4):437.

6. Garland EJ. Facing the evidence: antidepressant treatment in children and adolescents [editorial]. CMA7 2004;170(4):489-91.

Competing interests: None declared.

DOI:10.1503/cmaj.1040396

\section{[Three of the authors respond:]}

W e wish to respond specifically to Mark Voysey's point that industry bias in reporting trial results is probable but that such bias has been refuted by an employee of a multinational drug company.

In our study, we found that industryfunded trials are associated with positive results in both medical and surgical randomized trials. ${ }^{1}$ Hirsch, ${ }^{2}$ an industry representative, argues against that finding. He states that Merck trial protocols undergo extensive scrutiny both internally and by external regulatory bodies and committees. Indeed, our review of trials found that drug trials achieved significantly higher mean quality scores than surgical trials $(15.7 \mathrm{v} .13 .4$ out of a maximum of 21 points, $p<0.01$ ). Companies such as Merck should be commended for their policy to publish the results of all "hypothesis-testing" clinical trials regardless of their outcomes. However, available evidence suggests that this policy is not universally applied by all drug companies. As reported in our study, ${ }^{1}$ there is a large body of literature (1572 randomized trials) for which there is a greater than 2-fold odds of a positive study result in industry-sponsored trials compared with non-industry-sponsored trials (odds ratio 2.3). We do not believe that this is, as Hirsch characterizes it, a "relation ... of modest degree." Ultimately, readers must critically examine all published trials with specific focus on the nature of industry involvement.

\section{Mohit Bhandari \\ Victor M. Montori \\ P.J. Devereaux}

Department of Clinical Epidemiology

and Biostatistics

McMaster University

Hamilton, Ont.

\section{References}

1. Bhandari M, Busse JW, Jackowski D, Montori VM, Schünemann H, Sprague S, et al. Association between industry funding and statistically significant pro-industry findings in medical and surgical randomized trials. CMA7 2004;170(4):477-80.

2. Hirsch L. Randomized clinical trials: What gets published, and when? [editorial]. CMAJ 2004;170 (4):481-3.

Competing interests: None declared.

DOI:10.1503/cmaj.1040683

\section{[Dr. Herxheimer and Dr. Mintzes respond:]}

Ves, we think that Mark Voysey has indeed got it straight, although we believe that it is not appropriate to prescribe SSRIs for children or adolescents even as a last resort. As outlined in our commentary, ${ }^{1}$ there is just no convincing evidence that they work. Jureidini and associates ${ }^{2}$ have pointed out that even among the minority of trials that have been published, authors consistently exaggerated benefits and downplayed serious risks. Overall, these authors concluded that trial results failed to support a benefit of sufficient magnitude to outweigh the risks. ${ }^{2}$

Voysey tries never to complain without offering some constructive suggestion. In a similar spirit, we suggest that the changes needed are systemic. Canada's parliamentary health committee has just released an excellent report, ${ }^{3}$ calling for 3 key changes to drug regulation: better monitoring and public access to information on clinical trials, conditional drug approvals coupled with improved post-market surveillance and enforcement of Canada's law prohibiting direct-to-consumer ad- 